\title{
Applications of Matrices to a Matroidal Structure of Rough Sets
}

\author{
Jingqian Wang and William Zhu \\ Lab of Granular Computing, Minnan Normal University, Zhangzhou 363000, China \\ Correspondence should be addressed to William Zhu; williamfengzhu@gmail.com \\ Received 12 June 2013; Revised 26 September 2013; Accepted 10 October 2013 \\ Academic Editor: Francisco Chiclana
}

Copyright (C) 2013 J. Wang and W. Zhu. This is an open access article distributed under the Creative Commons Attribution License, which permits unrestricted use, distribution, and reproduction in any medium, provided the original work is properly cited.

\begin{abstract}
Rough sets provide an efficient tool for dealing with the vagueness and granularity in information systems. They are widely used in attribute reduction in data mining. There are many optimization issues in attribute reduction. Matroids generalize the linear independence in vector spaces and are widely used in optimization. Therefore, it is necessary to integrate rough sets and matroids. In this paper, we apply matrices to a matroidal structure of rough sets through three sides, which are characteristics, operations, and axioms. Firstly, a matroid is induced by an equivalence relation, and the matroid is a representable matroid whose representable matrix is a matrix representation of the equivalence relation. Then some characteristics of the matroid are presented through the representable matrix mainly. Secondly, contraction and restriction operations are applied to the matroid through the representable matrix and approximation operators of rough sets. Finally, two axioms of circuit incidence matrices of 2-circuit matroids are obtained, where 2-circuit matroids are proposed based on the characteristics of the matroid. In a word, these results show an interesting view to investigate the combination between rough sets and matroids through matrices.
\end{abstract}

\section{Introduction}

Rough set theory was proposed by Pawlak [1, 2] in 1982 as a tool to conceptualize, organize, and analyze various types of data in data mining. It has been widely used to deal with many practical problems, such as attribute reduction $[3,4]$, feature selection [5-7], rule extraction [8-11], and knowledge discovery [12-14]. Moreover, through extending equivalence relations or partitions, rough set theory has been extended to generalized rough sets based on relations $[15,16]$ and covering-based rough sets $[17,18]$.

However, many important problems including attribute reduction in rough sets are NP-hard. Therefore, the algorithms to solve them are often greedy ones [19, 20]. Matroid theory [21-23] is a generalization of linear algebra and graph theory and provides well-established platforms for greedy algorithms [24]. It has been used in diverse fields, such as combinatorial optimization [25], algorithm design [24], information coding [26], and cryptology [27]. Hence, matroid theory may bring new chances for rough set theory. Recently, matroid theory has been connected with other theories, such as rough set theory [28-31] and lattice theory $[32,33]$.
In this paper, we apply matrices to a matroidal structure of rough sets through three sides, which are characteristics, operations, and axioms. Firstly, a matroid is induced by an equivalence relation on a universe through the circuit axiom, and a matrix representation of the equivalence relation is a representable matrix of the matroid. Then some characteristics of the matroid, which are bases, the rank function, and circuits, are presented through the representable matrix of the matroid mainly. Secondly, contraction and restriction operations are applied to the matroid through the representable matrix and approximation operators of rough sets. We present some relationships between some new matroids, which are obtained by using contraction and restriction operations. For example, we present a relationship between these two matroids: one is the contraction of the matroid to the complement of a single point set; the other one is a vector matroid of a matrix obtained from the representable matrix by deleting all the columns whose labels are in the equivalence class of this point. Finally, the matroid belongs to a type of matroids, which is called 2-circuit matroid. Two axioms of circuit incidence matrices of 2-circuit matroids are obtained.

The rest of this paper is organized as follows. Section 2 reviews some fundamental definitions about rough sets and 
matroids. In Section 3, we prove that the matroid induced by an equivalence relation is a representable matroid, and some characteristics of the matroid are presented through the representable matrix of the matroid mainly. Then contraction and restriction operations are applied to the matroid through the representable matrix and approximation operators of rough sets. In Section 4 , the matroid belongs to a type of matroids called 2-circuit matroid. Two axioms of circuit incidence matrices of 2-circuit matroids are obtained. Finally, Section 5 concludes this paper and indicates further works.

\section{Basic Definitions}

This section recalls some fundamental definitions related to Pawlak's rough sets and matroids.

2.1. Pawlak's Rough Sets. Rough set theory provides a systematic approach to data preprocessing in data mining. In information or decision systems, any attribute subset is characterized by an equivalence relation. The following definition shows that a universe together with an equivalence relation on it forms an approximation space.

Definition 1 (approximation space $[34,35]$ ). Let $U$ be a nonempty and finite set called universe and $R$ an equivalence relation on $U$. The ordered pair $(U, R)$ is called a Pawlak's approximation space.

In rough sets, a pair of approximation operators is used to describe an object. In the following definition, we introduce the pair of approximation operators.

Definition 2 (approximation operator $[34,35]$ ). Let $R$ be an equivalence relation on $U$. A pair of approximation operators $R_{*}, R^{*}: 2^{U} \rightarrow 2^{U}$, is defined as follows: for all $X \subseteq U$,

$$
\begin{gathered}
R_{*}(X)=\{x \in U: R N(x) \subseteq X\}, \\
R^{*}(X)=\{x \in U: R N(x) \bigcap X \neq \emptyset\},
\end{gathered}
$$

where $R N(x)=\{y \in U: x R y\}$. They are called the lower and upper approximation operators with respect to $R$, respectively.

In an approximation space, if a subset can be precisely described by an equivalence relation, then it is called a precise set; otherwise, it is called a rough set.

Definition 3 ( $R$-rough set $[34,35]$ ). Let $R$ be an equivalence relation on $U$. For all $X \subseteq U$, if $R_{*}(X)=R^{*}(X)$, then $X$ is called a $R$-precise set; otherwise, $X$ is called a $R$-rough set.

2.2. Matroids. Matroids draw heavily on both linear algebra and graphs for their motivation and notation. They have a variety of applications in combinatorial optimization, algorithm design, information coding, and cryptology. In the following definition, one of the most valuable definitions of matroids is presented from the viewpoint of independent sets.
Definition 4 (matroid [21, 23]). A matroid $M$ is an ordered pair $(U, \mathbf{I})$ where $U$ (the ground set) is a finite set and $\mathbf{I}$ (the family of independent sets) is a family of subsets of $U$ with the following properties:

(I1) $\emptyset \in \mathbf{I}$;

(I2) if $I \in \mathbf{I}$ and $I^{\prime} \subseteq I$, then $I^{\prime} \in \mathbf{I}$;

(I3) if $I_{1}, I_{2} \in \mathrm{I}$ and $\left|I_{1}\right|<\left|I_{2}\right|$, then there exists $e \in I_{2}-I_{1}$ such that $I_{1} \bigcup\{e\} \in \mathbf{I}$, where $|I|$ denotes the cardinality of $I$.

We shall often write $U(M)$ for $U$ and $\mathbf{I}(M)$ for $\mathbf{I}$, particularly when several matroids are being considered. A type of matroids is provided to show that matroids generalize the linear independence in vector spaces.

Proposition 5 (vector matroid $[21,23]$ ). Let $U$ be the set of column labels of a matrix $A=\left(a_{i j}\right)_{m \times n}$ over a field $F$ and $\mathbf{I}$ the family of subsets $X$ of $U$ for which the columns labelled by $X$ are linearly independent in the vector space $V(m, F)$. Then $(U, \mathbf{I})$ is a matroid, and it is called the vector matroid of $A$, denoted by $M[A]$.

Note that, the field used in this paper is real number field R. The transpose of a matrix $A$ is denoted by $A^{\top}$. In order to show that linear algebra is an original source of matroid theory, we present an example from the viewpoint of the linear independence in vector spaces.

Example 6. Let $A=\left(\begin{array}{cccc}a_{1} & a_{2} & a_{3} & a_{4} \\ 1 & 0 & 0 & -1 \\ 0 & 1 & 0 & -1 \\ 0 & 0 & 1 & 0\end{array}\right)$ and $U=\left\{a_{1}, a_{2}, a_{3}, a_{4}\right\}$ be the set of all column labels of $A$. We suppose that $I=\{X \subseteq U$ : the columns labelled by $X$ are linearly independent in $V(3, \mathbf{R})\}$; that is, $\mathbf{I}=\left\{\emptyset,\left\{a_{1}\right\},\left\{a_{2}\right\},\left\{a_{3}\right\},\left\{a_{4}\right\},\left\{a_{1}, a_{2}\right\},\left\{a_{1}, a_{3}\right\},\left\{a_{1}, a_{4}\right\}\right.$, $\left.\left\{a_{2}, a_{3}\right\},\left\{a_{2}, a_{4}\right\},\left\{a_{3}, a_{4}\right\},\left\{a_{1}, a_{2}, a_{3}\right\},\left\{a_{1}, a_{3}, a_{4}\right\},\left\{a_{2}, a_{3}, a_{4}\right\}\right\}$. Then $M=(U, \mathbf{I})$ is a vector matroid, denoted by $M[A]$.

A type of matroids named representable matroids is defined through vector matroids.

Definition 7 (representable matroid [21, 23]). Let $M$ be a matroid. $M$ is called representable matroid if there exist a field $F$ and a matrix $A$ over $F$ such that $M=M[A] . A$ is called a representable matrix of $M$.

If a subset of the ground set is not an independent set of a matroid, then it is called a dependent set of the matroid. Based on the dependent set, we introduce the circuit of a matroid. For this purpose, some denotations are presented.

Definition 8 (see $[21,23]$ ). Let $\mathbf{A}$ be a family of subsets of $U$. One can denote the following:

$$
\begin{aligned}
& \operatorname{Low}(\mathbf{A})=\{X \subseteq U: \exists A \in \mathbf{A}(X \subseteq A)\} ; \\
& \operatorname{Min}(\mathbf{A})=\{X \in \mathbf{A}: \forall Y \in \mathbf{A}, Y \subseteq X \Rightarrow X=Y\} ; \\
& \operatorname{Opp}(\mathbf{A})=\{X \subseteq U: X \notin \mathbf{A}\} .
\end{aligned}
$$

The dependent set of a matroid generalizes the linear dependence in vector spaces and the cycle in graphs. Any circuit of a matroid is a minimal dependent set. 
Definition 9 (circuit $[21,23])$. Let $M=(U, \mathbf{I})$ be a matroid. A minimal dependent set in $M$ is called a circuit of $M$, and we denote the family of all circuits of $M$ by $\mathrm{C}(M)$; that is, $\mathbf{C}(M)=\operatorname{Min}(\operatorname{Opp}(\mathbf{I}))$.

Example 10 (continued from Example 6). $\mathrm{C}(M)=\operatorname{Min}(\{X \subseteq$ $U$ : the columns labelled by $X$ are linearly dependent in $V(3, \mathbf{R})\})$; that is, $\mathbf{C}(M)=\left\{\left\{a_{1}, a_{2}, a_{4}\right\}\right\}$.

The following proposition shows that a matroid can be defined from the viewpoint of circuits.

Proposition 11 (circuit axiom [21, 23]). Let $\mathbf{C}$ be a family of subsets of $U$. Then there exists a matroid $M$ such that $\mathbf{C}=$ $\mathbf{C}(M)$ if and only if $\mathbf{C}$ satisfies the following three conditions:

(C1) $\emptyset \notin \mathrm{C}$;

(C2) if $C_{1}, C_{2} \in \mathbf{C}$ and $C_{1} \subseteq C_{2}$, then $C_{1}=C_{2}$;

(C3) if $C_{1}, C_{2} \in \mathrm{C}, C_{1} \neq C_{2}$, and $x \in C_{1} \cap C_{2}$, then there exists $C_{3} \in \mathbf{C}$ such that $C_{3} \subseteq\left(C_{1} \cup C_{2}\right)-\{x\}$.

The following definition shows that a base of a matroid is a maximal independent set.

Definition 12 (base $[21,23])$. Let $M=(U, \mathbf{I})$ be a matroid. A maximal independent set in $M$ is called a base of $M$, and we denote the family of all bases of $M$ by $\mathbf{B}(M)$; that is, $\mathbf{B}(M)=$ $\operatorname{Max}(\mathbf{I})$.

The following proposition shows that a matroid can be determined by its bases.

Proposition 13 (base axiom [21, 23]). Let $\mathbf{B}$ be a family of subsets of $U$. Then there exists a matroid $M$ such that $\mathbf{B}=$ $\mathbf{B}(M)$ if and only if $\mathbf{B}$ satisfies the following two conditions:

(B1) $\mathbf{B} \neq \emptyset$;

(B2) if $B_{1}, B_{2} \in \mathbf{B}$ and $x \in B_{1}-B_{2}$, then there exists an element $y \in B_{2}-B_{1}$ such that $\left(B_{1}-\{x\}\right) \cup\{y\} \in \mathbf{B}$.

The rank of a matrix and the dimension of a vector space are important concepts in linear algebra. The following definition is a generalization of these two concepts.

Definition 14 (rank function $[21,23])$. Let $M=(U, I)$ be a matroid. The rank function $r_{M}$ of $M$ is defined as $r_{M}(X)=$ $\max \{|I|: I \subseteq X, I \in \mathbf{I}\}$ for all $X \subseteq U . r_{M}(X)$ is called the rank of $X$ in $M$.

\section{Matrix and the Matroid Induced by an Equivalence Relation}

In this section, we prove that the matroid induced by an equivalence relation is a representable matroid, and some characteristics of the matroid are presented through a representable matrix of the matroid mainly. Then contraction and restriction operations are applied to the matroid through the representable matrix and approximation operators of rough sets.
3.1. Characteristics of the Matroid through Matrices. In this subsection, a matroid is induced by an equivalence relation on a universe, and a matrix representation of the equivalence relation is a representable matrix of the matroid. Some characteristics of the matroid are presented through the representable matrix mainly. The following proposition shows that the matroid can be induced through the circuit axiom.

Proposition 15 (see [29]). Let $R$ be an equivalence relation on $U$. One has that

$$
\{\{x, y\} \subseteq U: x \neq y,(x, y) \in R\}
$$

satisfies (C1), (C2), and (C3) of Proposition 11. Then there exists a matroid $M$ such that $\mathbf{C}(M)=\{\{x, y\} \subseteq U$ : $x \neq y,(x, y) \in R\}$, and we denote this matroid by $M(R)$.

In order to find a matrix to prove that the matroid induced by an equivalence relation is a representable matroid, a matrix representation of an equivalence relation is presented in the following definition.

Definition 16. Let $R$ be an equivalence relation on $U=$ $\left\{x_{1}, x_{2}, \ldots, x_{n}\right\}$ and a partition $U / R=\left\{P_{1}, P_{2}, \ldots, P_{m}\right\}$. We define a Boolean matrix $A_{R}=\left(a_{i j}\right)_{m \times n}$ as follows:

$$
a_{i j}= \begin{cases}1, & x_{j} \in P_{i}, \\ 0, & x_{j} \notin P_{i} .\end{cases}
$$

$x_{j}$ labels the $j$ th column and $P_{i}$ labels the $i$ th row. $A_{R}$ is called a matrix representation of $R$.

Note that $a_{i j}$ is 1 or 0 depending on whether $x_{j}$ is or is not in $P_{i}$. An equivalence relation on a universe may have different matrix representations because of the randomness of the order of a set.

Example 17. Let $U=\{a, b, c\}$ and $U / R=\{\{a\},\{b, c\}\}$. Then $A_{R}=\left\{\begin{array}{c}\{a\} \\ \{b, c\}\end{array} \quad\left(\begin{array}{lll}a & b & c \\ 1 & 0 & 0 \\ 0 & 1 & 1\end{array}\right), A_{R}^{\prime}=\left\{\begin{array}{c}\{, c\} \\ \{a\}\end{array}\left(\begin{array}{lll}a & b & c \\ 0 & 1 & 1 \\ 1 & 0 & 0\end{array}\right)\right.\right.$, and $A_{R}^{\prime \prime}=\begin{array}{c}\{a\} \\ b b, c\}\end{array}\left(\begin{array}{lll}b & a & c \\ 0 & 1 & 0 \\ 1 & 0 & 1\end{array}\right)$ are some matrix representations of $R$.

On one hand, according to Definition 16, two matrix representations of $R$ with different orders of column labels have the same vector matroid because the columns labelled by $X \subseteq$ $U$ in these two matrices have the same linear dependence. On the other hand, there is an important result in matroid theory that a matrix and its row elementary transformed matrix have the same vector matroid. Therefore, all matrix representations of $R$ induce the same vector matroid.

Example 18 (continued from Example 17). $M\left[A_{R}\right]=(U$, $\mathbf{I}\left(M\left[A_{R}\right]\right)$, where $\mathbf{I}\left(M\left[A_{R}\right]\right)=\operatorname{Low}(\{\{a, b\},\{a, c\}\}) ; M\left[A_{R}^{\prime}\right]=$ $\left(U, \mathbf{I}\left(M\left[A_{R}^{\prime}\right]\right)\right)$, where $\mathbf{I}\left(M\left[A_{R}^{\prime}\right]\right)=\mathbf{I}\left(M\left[A_{R}\right]\right) ; M\left[A_{R}^{\prime \prime}\right]=(U$, $\left.\mathbf{I}\left(M\left[A_{R}^{\prime \prime}\right]\right)\right)$, where $\mathbf{I}\left(M\left[A_{R}^{\prime \prime}\right]\right)=\mathbf{I}\left(M\left[A_{R}\right]\right)$. Therefore, $M\left[A_{R}\right]=M\left[A_{R}^{\prime}\right]=M\left[A_{R}^{\prime \prime}\right]$.

The following proposition shows that the matroid induced by an equivalence relation is a representable matroid, and a matrix representation of the equivalence relation is a representable matrix of the matroid. 
Proposition 19. Let $R$ be an equivalence relation on $U=$ $\left\{x_{1}, x_{2}, \ldots, x_{n}\right\}$ and $U / R=\left\{P_{1}, P_{2}, \ldots, P_{m}\right\}$. Then $M(R)=$ $M\left[A_{R}\right]$.

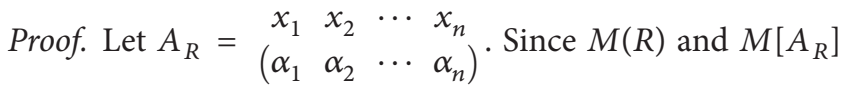
have the same ground set, so we need to prove only that $\mathbf{C}(M(R))=\mathbf{C}\left(M\left[A_{R}\right]\right)$. According to Proposition 5 and Definition 9, $\mathbf{C}\left(M\left[A_{R}\right]\right)=\operatorname{Min}(\{C \subseteq U$ : the columns labelled by $C$ are linearly dependent in $V(m, \mathbf{R})\})$. According to Definition 16, $\alpha_{i} \neq(0 \cdots 0)^{\top}$. Hence, $\left\{x_{i}\right\} \notin \mathbf{C}\left(M\left[A_{R}\right]\right)$ for all $i \in\{1,2, \ldots, n\}$. For any $\left\{x_{i}, x_{j}\right\} \in \mathbf{C}(M(R)),\left(x_{i}, x_{j}\right) \in$ $R$. According to Definition 16, the columns labelled by $x_{i}$ and $x_{j}$ are the same. Therefore, the columns labelled by $\left\{x_{i}, x_{j}\right\}$ are linearly dependent in $V(m, \mathbf{R})$. Hence, $\left\{x_{i}, x_{j}\right\} \in$ $\mathbf{C}\left(M\left[A_{R}\right]\right)$; that is, $\mathbf{C}(M(R)) \subseteq \mathbf{C}\left(M\left[A_{R}\right]\right)$. Conversely, if $C \in$ $\mathbf{C}\left(M\left[A_{R}\right]\right)-\mathbf{C}(M(R))$, according to $(C 2)$ of Proposition 11, $\{x, y\} \nsubseteq C$ for any $\{x, y\} \in \mathbf{C}(M(R))$. Therefore, for any $x_{i}, x_{j} \in C$ and $x_{i} \neq x_{j},\left(x_{i}, x_{j}\right) \notin R$. According to Definition 16, there is only a 1 in every column of $A_{R}$ and the columns labelled by $C$ are different. Hence, the columns labelled by $C$ are linearly independent in $V(m, \mathbf{R})$, which is contradictory with $C \in \mathbf{C}\left(M\left[A_{R}\right]\right)$. Thus $\mathbf{C}\left(M\left[A_{R}\right]\right) \subseteq$ $\mathrm{C}(M(R))$. This completes the proof.

Using the representable matrix of the matroid induced by an equivalence relation, some characteristics of the matroid will be obtained. For this purpose, the following definition proposes a function from a power set of a set to a set of $n$ dimensional $0-1$ vectors.

Definition 20 (see [36]). If $U=\left\{x_{1}, x_{2}, \ldots, x_{n}\right\}$ and $X \subseteq U$, then the characteristic function of $X$ is defined as $\varphi(X)=$ $\left(a_{1}, \ldots, a_{i}, \ldots, a_{n}\right)$, where

$$
a_{i}= \begin{cases}1, & x_{i} \in X, \\ 0, & x_{i} \notin X .\end{cases}
$$

As we know, $\varphi$ is a bijection from $2^{U}$ to the set of all $n$ dimensional 0-1 vectors. Let $A=\left(a_{i j}\right)_{m \times n}$ and $B=\left(b_{i j}\right)_{n \times l}$ be Boolean matrices. Then $A \odot B=\left(c_{i j}\right)_{m \times l}$, where $c_{i j}=\mathrm{V}_{s=1}^{n}\left(a_{i s} \wedge\right.$ $\left.b_{s j}\right)$ and $\vee, \wedge$ denote maximum and minimum, respectively. In this paper, $\odot$ denotes Boolean product of matrices and "." denotes multiplication of matrices.

The following proposition presents the family of bases of the matroid induced by an equivalence relation from the viewpoint of a representable matrix of the matroid.

Proposition 21. Let $R$ be an equivalence relation on $U$. Then $\mathbf{B}(M(R))=\operatorname{Min}\left(\left\{X \subseteq U: A_{R} \odot(\varphi(X))^{\top}=\mathbf{1}^{v}\right\}\right)$, where $\mathbf{1}^{v}$ is a column vector whose entries are all 1 .

Proof. For any $X \subseteq U$ and $A_{R} \odot(\varphi(X))^{\top}=\mathbf{1}^{v}$, we have $R N(x) \bigcap X \neq \emptyset$ for any $x \in U$. Therefore, $\operatorname{Min}(\{X \subseteq U$ : $\left.\left.A_{R} \odot(\varphi(X))^{\top}=\mathbf{1}^{v}\right\}\right)=\operatorname{Min}(\{X \subseteq U: \forall x \in$ $U, R N(x) \bigcap X \neq \emptyset\})$. According to Definition 8, $\operatorname{Min}(\{X \subseteq$ $U: \forall x \in U, R N(x) \bigcap X \neq \emptyset\})=\{X \subseteq U: \forall x \in$ $U,|R N(x) \bigcap X|=1\}$. First of all, we should prove that $\{X \subseteq$ $U: \forall x \in U,|R N(x) \bigcap X|=1\}$ satisfies (B1) and (B2) of Proposition 13.
(B1) : It is straightforward that $\{X \subseteq U: \forall x \in$ $U,|R N(x) \bigcap X|=1\} \neq \emptyset$.

(B2) : If $B_{1}, B_{2} \in\{X \subseteq U: \forall x \in U,|R N(x) \bigcap X|=1\}$ and $s \in B_{1}-B_{2}$,

then there exists $y \in B_{2}-B_{1}$ and $y \in R N(s)$. Since $B_{1} \bigcap R N(s)=\{s\}$, so $\left(\left(B_{1}-\{s\}\right) \bigcup\{y\}\right) \bigcap R N(s)=\{y\}$. For any $z \in U-R N(s),\left|\left(\left(B_{1}-\{s\}\right) \bigcup\{y\}\right) \bigcap R N(z)\right|=1$. Therefore, $\left(\left(B_{1}-\{s\}\right) \bigcup\{y\}\right) \in\{X \subseteq U: \forall x \in U,|R N(x) \bigcap X|=1\}$.

Hence, $\{X \subseteq U: \forall x \in U,|R N(x) \cap X|=1\}$ satisfies (B1) and (B2) of Proposition 13. Then we should prove that the matroid whose family of bases is $\{X \subseteq U$ : $\forall x \in U,|R N(x) \bigcap X|=1\}$ is $M(R)$; that is, they have the same family of circuits. Since $\operatorname{Low}(\{X \subseteq U: \forall x \in$ $U,|R N(x) \bigcap X|=1\})=\{X \subseteq U: \forall x \in U,|R N(x) \bigcap X| \leq$ $1\}$, so $\operatorname{Min}(\operatorname{Opp}(\{X \subseteq U: \forall x \in U,|R N(x) \bigcap X| \leq 1\}))=$ $\operatorname{Min}(\{X \subseteq U: \exists x \in U,|R N(x) \cap X| \geq 2\})=\{\{x, y\} \subseteq$ $R N(x): x \neq y\}=\mathbf{C}(M(R))$. Hence, $\mathbf{B}(M(R))=\operatorname{Min}(\{X \subseteq$ $\left.\left.U: A_{R} \odot(\varphi(X))^{\top}=\mathbf{1}^{v}\right\}\right)$. This completes the proof.

According to Proposition 21, a representable matrix of the matroid induced by an equivalence relation and the corresponding characteristic function can get the family of bases of the matroid. In order to get the family of bases of the matroid, we need to get only one representable matrix.

Example 22 (continued from Example 17). $A_{R} \odot(\varphi(\{a\}))^{\top}=$ $\left(\begin{array}{lll}1 & 0 & 0 \\ 0 & 1 & 1\end{array}\right) \odot\left(\begin{array}{lll}1 & 0 & 0\end{array}\right)^{\top}=\left(\begin{array}{lll}1 & 0 & 0 \\ 0 & 1 & 1\end{array}\right) \odot\left(\begin{array}{l}1 \\ 0 \\ 0\end{array}\right)=\left(\begin{array}{l}1 \\ 0\end{array}\right) ; A_{R} \odot(\varphi(\{b\}))^{\top}=$ $A_{R} \odot\left(\begin{array}{lll}0 & 1 & 0\end{array}\right)^{\top}=\left(\begin{array}{l}0 \\ 1\end{array}\right) ; A_{R} \odot(\varphi(\{c\}))^{\top}=\left(\begin{array}{l}0 \\ 1\end{array}\right) ; A_{R} \odot$ $(\varphi(\{a, b\}))^{\top}=\left(\begin{array}{l}1 \\ 1\end{array}\right) ; A_{R} \odot(\varphi(\{a, c\}))^{\top}=\left(\begin{array}{l}1 \\ 1\end{array}\right) ; A_{R} \odot$ $(\varphi(\{b, c\}))^{\top}=\left(\begin{array}{l}0 \\ 1\end{array}\right) ; A_{R} \odot(\varphi(\{a, b, c\}))^{\top}=\left(\begin{array}{l}1 \\ 1\end{array}\right)$. Hence, $\mathbf{B}(M(R))=\operatorname{Min}(\{\{a, b\},\{a, c\},\{a, b, c\}\})=\{\{a, b\},\{a, c\}\}$.

The following proposition presents another method to obtain the family of bases of the matroid induced by an equivalence relation from the viewpoint of a representable matrix.

Proposition 23. Let $R$ be an equivalence relation on $U=$ $\left\{x_{1}, x_{2}, \ldots x_{n}\right\}, U / R=\left\{P_{1}, P_{2}, \ldots, P_{m}\right\}$, and $A_{R}=\left(a_{i j}\right)_{m \times n}$. Then $\mathbf{B}(M(R))=\left\{\left\{x_{j_{1}}, x_{j_{2}}, \ldots, x_{j_{m}}\right\}: a_{1 j_{1}} a_{2 j_{2}} \cdots a_{m j_{m}}=1\right\}$.

Proof. According to the proof of Proposition 21, $\mathbf{B}(M(R))=$ $\{X \subseteq U: \forall x \in U,|R N(x) \cap X|=1\}$. We need to prove only $\left\{\left\{x_{j_{1}}, x_{j_{2}}, \ldots, x_{j_{m}}\right\}: a_{1 j_{1}} a_{2 j_{2}} \cdots a_{m j_{m}}=1\right\}=\{X \subseteq U$ : $\forall x \in U,|R N(x) \bigcap X|=1\}$. For all $X \in\left\{\left\{x_{j_{1}}, x_{j_{2}}, \ldots, x_{j_{m}}\right\}\right.$ : $\left.a_{1 j_{1}} a_{2 j_{2}} \cdots a_{m j_{m}}=1\right\}$, suppose that $X=\left\{x_{j_{1}}, x_{j_{2}}, \ldots, x_{j_{m}}\right\}$. Since $a_{1 j_{1}} a_{2 j_{2}} \cdots a_{m j_{m}}=1$, so $a_{i j_{i}}=1$ for any $1 \leq i \leq m$. Therefore, $x_{j_{i}} \in P_{i}$; that is, $|X \bigcap R N(x)|=1$ for any $x \in U$. Hence, $\left\{\left\{x_{j_{1}}, x_{j_{2}}, \ldots, x_{j_{m}}\right\}: a_{1 j_{1}} a_{2 j_{2}} \cdots a_{m j_{m}}=1\right\} \subseteq\{X \subseteq U$ : $\forall x \in U,|R N(x) \cap X|=1\}$. Conversely, for all $X \in\{X \subseteq$ $U: \forall x \in U,|R N(x) \bigcap X|=1\}$, it is straightforward that $X \in\left\{\left\{x_{j_{1}}, x_{j_{2}}, \ldots, x_{j_{m}}\right\}: a_{1 j_{1}} a_{2 j_{2}} \cdots a_{m j_{m}}=1\right\}$. Therefore, $\{X \subseteq U: \forall x \in U,|R N(x) \bigcap X|=1\} \subseteq\left\{\left\{x_{j_{1}}, x_{j_{2}}, \ldots, x_{j_{m}}\right\}:\right.$ $\left.a_{1 j_{1}} a_{2 j_{2}} \cdots a_{m j_{m}}=1\right\}$. This completes the proof.

The following definition presents a matrix related to circuits of a matroid. 
Definition 24 (circuit incidence matrix [21, 23]). Let $M$ be a matroid on $U=\left\{x_{1}, x_{2}, \ldots, x_{n}\right\}$ and $\mathrm{C}(M)=$ $\left\{C_{1}, C_{2}, \ldots, C_{m}\right\}$. A circuit incidence matrix of $M$ is $A(\mathbf{C}(M))=\left(a_{i j}\right)_{m \times n}$, where

$$
a_{i j}= \begin{cases}1, & x_{j} \in C_{i}, \\ 0, & x_{j} \notin C_{i} .\end{cases}
$$

According to Definition 24, circuits can be replaced by bases of a matroid. Therefore, we can also get a base incidence matrix. Let $M$ be a matroid on $U=\left\{x_{1}, x_{2}, \ldots, x_{n}\right\}$ and $\mathbf{B}(M)=\left\{B_{1}, B_{2}, \ldots, B_{m}\right\}$. A base incidence matrix of $M$ is $A(\mathbf{B}(M))=\left(a_{i j}\right)_{m \times n}$, where

$$
a_{i j}= \begin{cases}1, & x_{j} \in B_{i}, \\ 0, & x_{j} \notin B_{i} .\end{cases}
$$

Note that the matroid induced by an equivalence relation may have different base incidence matrices because of the randomness of the order of a set. But these base incidence matrices can induce a unique rank function of the matroid.

Proposition 25. Let $R$ be an equivalence relation on $U$ and $X \subseteq U$. Then $r_{M(R)}(X)=\max _{1 \leq i \leq l} b_{i}$, where

$$
\left(\begin{array}{c}
b_{1} \\
\vdots \\
b_{i} \\
\vdots \\
b_{l}
\end{array}\right)=A(\mathbf{B}(M(R))) \cdot \varphi(X)^{\top} .
$$

Proof. It is straightforward that $b_{i}=\left|B_{i} \cap X\right|$, where $B_{i} \in$ $\mathbf{B}(M(R))$. Suppose that $b_{j}=\max _{1 \leq i \leq l} b_{i}$. Since $B_{j} \in \mathbf{B}(M(R))$, so $B_{j} \cap X \in \mathbf{I}(M(R))$. In fact, if there exists $I_{1} \in \mathbf{I}(M(R))$ such that $\left|I_{1} \cap X\right|>\left|B_{j} \cap X\right|$, then there must be a $B_{k} \in \mathbf{B}(M(R))$ such that $I_{1} \subseteq B_{k}$. Therefore, $b_{k}=\left|B_{k} \cap X\right| \geq\left|I_{1} \cap X\right|>$ $\left|B_{j} \cap X\right|=b_{j}$, which is contradictory with $b_{j}=\max _{1 \leq i \leq l} b_{i}$. Hence, $r_{M(R)}(X)=\max _{1 \leq i \leq l} b_{i}$. This completes the proof.

Example 26 (continued from Example 22). Let $X_{1}=\{a, b\}$, and $X_{2}=\{b, c\}, A(\mathbf{B}(M(R)))=\left(\begin{array}{ccc}a & b & c \\ 1 & 1 & 0 \\ 1 & 0 & 1\end{array}\right)$. Since $A(\mathbf{B}(M(R)))$. $\varphi\left(X_{1}\right)^{\top}=\left(\begin{array}{lll}1 & 1 & 0 \\ 1 & 0 & 1\end{array}\right) \cdot\left(\begin{array}{l}1 \\ 1 \\ 0\end{array}\right)=\left(\begin{array}{l}2 \\ 1\end{array}\right)$, so $r_{M(R)}\left(X_{1}\right)=2$; since $A(\mathbf{B}(M(R))) \cdot \varphi\left(X_{2}\right)^{\top}=\left(\begin{array}{l}1 \\ 1\end{array}\right)$, so $r_{M(R)}\left(X_{2}\right)=1$.

The rank function of the matroid induced by an equivalence relation can also be obtained through the representable matrix of the matroid.

Proposition 27. Let $R$ be an equivalence relation on $U$ and $X \subseteq U$. Then $r_{M(R)}(X)=\mathbf{1}_{v} \cdot\left(A_{R} \odot \varphi(X)^{\top}\right)$, where $\mathbf{1}_{v}$ is a row vector whose entries are all 1 .

Proof. Suppose that $U / R=\left\{P_{1}, P_{2}, \ldots, P_{m}\right\}$. The $i$ th entry of $A_{R} \odot \varphi(X)$ is 1 or 0 depending on whether or not $P_{i} \cap X \neq \emptyset$. It is straightforward that $\mathbf{1}_{v} \cdot\left(A_{R} \odot \varphi(X)^{\top}\right)=$ $|\{R N(x): x \in U, R N(x) \bigcap X \neq \emptyset\}|$. We need to prove only that $r_{M(R)}(X)=|\{R N(x): x \in U, R N(x) \bigcap X \neq \emptyset\}|$.
According to Proposition 25, $r_{M(R)}(X)=\max \{|B \bigcap X|$ : $B \in \mathbf{B}(M(R))\}$. According to the proof of Proposition 21, $\mathbf{B}(M(R))=\{X \subseteq U: \forall x \in U,|R N(x) \bigcap X|=1\}$. Therefore, $r_{M(R)}(X)=|\{R N(x): x \in U, R N(x) \cap X \neq \emptyset\}|$. This completes the proof.

Example 28 (continued from Example 26). $r_{M(R)}\left(X_{1}\right)=\mathbf{1}_{v}$. $\left(A_{R} \odot \varphi\left(X_{1}\right)^{\top}\right)=\left(\begin{array}{ll}1 & 1\end{array}\right) \cdot\left(\left(\begin{array}{lll}1 & 1 & 0 \\ 1 & 0 & 1\end{array}\right) \odot\left(\begin{array}{l}1 \\ 1 \\ 0\end{array}\right)\right)=\left(\begin{array}{ll}1 & 1\end{array}\right) \cdot\left(\begin{array}{l}1 \\ 1\end{array}\right)=2$.

Propositions 25 and 27 present two direct and simple methods to calculate the rank function of the matroid induced by an equivalence relation compared with the traditional method in Definition 14. In fact, the rank function of the matroid can be used in attribute reduction of information systems. Integrating these two methods with the following theorem which presents an equivalent formulation of attribute reduction of information systems, one can obtain a reduct of an information system.

Theorem 29 (see [29]). Let $I S=(U, A)$ be an information system. For all $B \subseteq A, B$ is a reduct of IS if and only if it satisfies the following two conditions:

(1) $\forall b \in B,\left\{X \subseteq U: \forall c \in B-\{b\}, r_{M(c)}(X)=1\right\} \neq\{X \subseteq$ $\left.U: \forall c \in B, r_{M(c)}(X)=1\right\}$;

(2) $\left\{X \subseteq U: \forall b \in B, r_{M(b)}(X)=1\right\}=\{X \subseteq U: \forall a \in$ $\left.A, r_{M(a)}(X)=1\right\}$,

where $M(a)$ denotes the matroid induced by the equivalence relation induced by attribute subset $\{a\}$.

In order to present circuits of the matroid induced by an equivalence relation from the viewpoint of matrices, a new matrix is established through a matrix representation of an equivalence relation and its transpose.

Lemma 30. Let $R$ be an equivalence relation on $U=\left\{x_{1}\right.$, $\left.x_{2}, \ldots, x_{n}\right\}$. Then $A_{R}^{\top} \cdot A_{R}=\left(b_{i j}\right)_{n \times n}$ is a relational matrix of $R$, where

$$
b_{i j}= \begin{cases}1, & \left(x_{i}, x_{j}\right) \in R \\ 0, & \text { otherwise. }\end{cases}
$$

Note that the matrix representation of an equivalence relation is the representable matrix of the matroid induced by the equivalence relation. Therefore, the family of circuits of the matroid can be obtained through the representable matrix. Let $\mathbf{e}_{i}$ be a $n$-dimensional 0 - 1 vector in which only the $i$ th entry is 1 ; that is, $\mathbf{e}_{i}=(0, \ldots, 0,1,0, \ldots, 0)$.

Proposition 31. Let $R$ be an equivalence relation on $U$ and $U=\left\{x_{1}, x_{2}, \ldots, x_{n}\right\}$. Then $\mathbf{C}(M(R))=\left\{\left\{x_{i}, x_{j}\right\}: \mathbf{e}_{i} \cdot\left(A_{R}^{\top}\right.\right.$. $\left.\left.A_{R}\right) \cdot \mathbf{e}_{j}^{\top}=1(1 \leq i<j \leq n)\right\}$.

Proof. We need to prove only that $\left\{\left\{x_{i}, x_{j}\right\}: \mathbf{e}_{i} \cdot\left(A_{R}^{\top} \cdot A_{R}\right) \cdot \mathbf{e}_{j}^{\top}=\right.$ $1(1 \leq i<j \leq n)\}=\left\{\left\{x_{i}, x_{j}\right\} \subseteq U: x_{i} \neq x_{j},\left(x_{i}, x_{j}\right) \in R\right\}$. Let $A_{R}^{\top} \cdot A_{R}=\left(b_{i j}\right)_{n \times n}$. For any $1 \leq i<j \leq n, \mathbf{e}_{i} \cdot\left(A_{R}^{\top} \cdot A_{R}\right) \cdot \mathbf{e}_{j}^{\top}=b_{i j}$. According to Lemma $30, b_{i j}=1$ if and only if $\left(x_{i}, x_{j}\right) \in R$. Since $R$ is an equivalence relation, so $A_{R}^{\top} \cdot A_{R}$ is a symmetric matrix. Therefore, $\left\{\left\{x_{i}, x_{j}\right\}: \mathbf{e}_{i} \cdot\left(A_{R}^{\mathrm{T}} \cdot A_{R}\right) \cdot \mathbf{e}_{j}^{\top}=1(1 \leq i<\right.$ 
$j \leq n)\}=\left\{\left\{x_{i}, x_{j}\right\}:\left(x_{i}, x_{j}\right) \in R(1 \leq i<j \leq n)\right\}=\left\{\left\{x_{i}, x_{j}\right\} \subseteq\right.$ $\left.U: x_{i} \neq x_{j},\left(x_{i}, x_{j}\right) \in R\right\}$. This completes the proof.

Example 32 (continued from Example 22). There is $A_{R}^{\top} \cdot A_{R}$

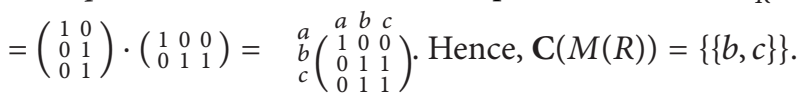

3.2. Contraction and Restriction Operations through Matrices. This subsection applies contraction and restriction operations to the matroid induced by an equivalence relation through the representable matrix and approximation operators of rough sets. First of all, the concepts of these two operations are presented in the following two propositions. In fact, two new matroids are obtained by applying restriction and contraction operations to a matroid, respectively.

Proposition 33 (restriction and deletion [21, 23]). Let $M=$ $(U, \mathbf{I})$ be a matroid and $X \subseteq U$. Then $\left(X, \mathbf{I}_{X}\right)$ is a matroid, where $\mathbf{I}_{X}=\{I \subseteq X: I \in \mathbf{I}\}$. We call this matroid the restriction of $M$ to $X$ and denote it by $M \mid X . M \backslash X=\left(U-X, \mathbf{I}_{U-X}\right)$ is called the deletion of $U-X$ from $M$.

Proposition 34 (contraction [21, 23]). Let $M=(U, I)$ be a matroid and $X \subseteq U$ and $B_{X}$ is a base of $M \mid X$ (i.e., $B_{X} \in$ $\mathbf{B}(M \mid m X))$. Then $\left(U-X, \mathbf{I}^{\prime}\right)$ is matroid, where $\mathbf{I}^{\prime}=\{I \subseteq U-X$ : $\left.I \cup B_{X} \in \mathbf{I}\right\}$. We call this matroid the contraction of $M$ to $U-X$ and denote it by $M / X$.

Note that the definition of $M / X$ has no relationship with the selection of $B_{X} \in \mathbf{B}(M \mid X)$. The following lemma shows an interesting relationship between a single point set and the equivalence class of this point through applying contraction and restriction operations to the matroid induced by an equivalence relation.

Lemma 35 (see [37]). Let $R$ be an equivalence relation on $U$. For all $x \in U, \mathbf{I}(M(R) /\{x\})=\mathbf{I}(M(R) / R N(x))=\mathbf{I}(M(R) \backslash$ $R N(x))$.

Let $A$ be a matrix over $F$ and $X$ a subset of the set $U$ of column labels of $A$. We shall denote by $A \ominus X$ the matrix obtained from $A$ by deleting all the columns whose labels are in $X$.

Inspired by Lemma 35, we obtain a relationship between two matroids, which are the contraction of the matroid induced by an equivalence relation to the complement of a single point set and a vector matroid of a matrix obtained from the representable matrix by deleting all the columns whose labels are in the equivalence class of this point.

Proposition 36. Let $R$ be an equivalence relation on $U$. Then $\mathbf{I}(M(R) /\{x\})=\mathbf{I}\left(M\left[A_{R} \ominus R N(x)\right]\right)$.

Proof. According to Proposition 19 and Lemma 35, we need to prove only that $\mathbf{I}\left(M\left[A_{R}\right] \backslash R N(x)\right)=\mathbf{I}\left(M\left[A_{R} \ominus R N(x)\right]\right)$. According to Proposition 33, $\mathbf{I}\left(M\left[A_{R}\right] \backslash R N(x)\right)=\{I \subseteq$ $\left.U\left(M\left[A_{R}\right]\right)-R N(x): I \in \mathbf{I}\left(M\left[A_{R}\right]\right)\right\}$. According to Proposition 5, $I \in \mathbf{I}\left(M\left[A_{R}\right] \backslash R N(x)\right)$ if and only if $I \subseteq$ $U\left(M\left[A_{R}\right]\right)-R N(x)$ and the columns labelled by $I$ are linearly independent. $I \in \mathbf{I}\left(M\left[A_{R} \ominus R N(x)\right]\right)$ if and only if $I \subseteq$ $U\left(M\left[A_{R} \ominus R N(x)\right]\right)$ and the columns labelled by $I$ are linearly independent. Since $U\left(M\left[A_{R}\right]\right)$ is the set of all columns of $A_{R}$ and $U\left(M\left[A_{R} \ominus R N(x)\right]\right)$ is the set of all columns of $A_{R} \ominus$ $R N(x)$, so $U\left(M\left[A_{R}\right]\right)-R N(x)=U\left(M\left[A_{R} \ominus R N(x)\right]\right)$. Hence, $\mathbf{I}\left(M\left[A_{R}\right] \backslash R N(x)\right)=\mathbf{I}\left(M\left[A_{R} \ominus R N(x)\right]\right)$. This completes the proof.

Note that the above proposition also shows a relationship between a single point set and the equivalence class of this point.

Example 37 (continued from Example 22). Since $R N(b)=$ $\{b, c\}$ and

$$
A_{R} \ominus R N(b)=\left(\begin{array}{ccc}
a & b & c \\
1 & 0 & 0 \\
0 & 1 & 1
\end{array}\right) \ominus\{b, c\}=\left(\begin{array}{l}
1 \\
0
\end{array}\right)
$$

so $\mathbf{I}\left(M\left[A_{R} \ominus R N(b)\right]\right)=\{\emptyset,\{a\}\}$. Since $\mathbf{B}(M(R))=\{\{a, b\}$, $\{a, c\}\}$, so $\mathbf{I}(M(R))=\operatorname{Low}(\mathbf{B}(M(R)))=\{\emptyset,\{a\},\{b\},\{c\}$, $\{a, b\},\{a, c\}\}, M(R) \mid\{b\}=\left(\{b\}, \mathbf{I}_{\{b\}}\right),\{b\} \in \mathbf{B}(M(R) \mid\{b\})$, where $\mathbf{I}_{\{b\}}=\{\emptyset,\{b\}\}$ and $\mathbf{B}(M(R) \mid\{b\})=\{\{b\}\}$. Hence, $\mathbf{I}(M(R) /\{b\})=\{\emptyset,\{a\}\}$. Therefore, $\mathbf{I}(M(R) /\{b\})=\mathbf{I}\left(M\left[A_{R} \ominus\right.\right.$ $R N(b)])$.

The following corollary presents an equivalent description of the above proposition from the viewpoint of upper approximations.

Corollary 38. Let $R$ be an equivalence relation on $U$. Then $\mathbf{I}(M(R) /\{x\})=\mathbf{I}\left(M\left[A_{R} \ominus R^{*}(\{x\})\right]\right)$.

When we apply a sequence of operations to the matroid induced by an equivalence relation like Lemma 35, the following lemma is obtained.

Lemma 39 (see [37]). Let $R$ be an equivalence relation on $U$. For all $X \subseteq U, \mathbf{I}(M(R) / X)=\mathbf{I}\left(M(R) / R^{*}(X)\right)=\mathbf{I}(M(R) \backslash$ $\left.R^{*}(X)\right)$.

Inspired by Proposition 36, we obtain the similar relationship between a subset and the upper approximation of this subset through these two operations and the representable matrix of the matroid induced by an equivalence relation.

Proposition 40. Let $R$ be an equivalence relation on $U$. Then $\mathbf{I}(M(R) / X)=\mathbf{I}\left(M\left[A_{R} \ominus R^{*}(X)\right]\right)$.

Proof. According to Proposition 19 and Lemma 39, we need to prove only that $\mathbf{I}\left(M\left[A_{R}\right] \backslash R^{*}(X)\right)=\mathbf{I}\left(M\left[A_{R} \ominus R^{*}(X)\right]\right)$. According to Proposition 33, $\mathbf{I}\left(M\left[A_{R}\right] \backslash R^{*}(X)\right)=\{I \subseteq$ $\left.U\left(M\left[A_{R}\right]\right)-R^{*}(X): I \in \mathbf{I}\left(M\left[A_{R}\right]\right)\right\}$. According to Proposition 5, $I \in \mathbf{I}\left(M\left[A_{R}\right] \backslash R^{*}(X)\right)$ if and only if $I \subseteq$ $U\left(M\left[A_{R}\right]\right)-R^{*}(X)$ and the columns labelled by $I$ are linearly independent. $I \in \mathbf{I}\left(M\left[A_{R} \ominus R^{*}(X)\right]\right)$ if and only if $I \subseteq$ $U\left(M\left[A_{R} \ominus R^{*}(X)\right]\right)$ and the columns labelled by $I$ are linearly independent. Since $U\left(M\left[A_{R}\right]\right)$ is the set of all columns of $A_{R}$ and $U\left(M\left[A_{R} \ominus R^{*}(X)\right]\right)$ is the set of all columns of $A_{R} \ominus$ $R^{*}(X)$, so $U\left(M\left[A_{R}\right]\right)-R^{*}(X)=U\left(M\left[A_{R} \ominus R^{*}(X)\right]\right)$. Hence, 
$\mathbf{I}\left(M\left[A_{R} \ominus R^{*}(X)\right]\right)=\mathbf{I}\left(M\left[A_{R}\right] \backslash R^{*}(X)\right)$. This completes the proof.

Example 41 (continued from Example 22). Let $X=\{b, c\}$. Since $R^{*}(X)=\{b, c\}$ and

$$
A_{R} \ominus R^{*}(X)=\left(\begin{array}{lll}
a & b & c \\
0 & 0 & 0 \\
0 & 1 & 1
\end{array}\right) \ominus\{b, c\}=\left(\begin{array}{l}
a \\
1 \\
0
\end{array}\right),
$$

so $\mathbf{I}\left(M\left[A_{R} \ominus R^{*}(X)\right]\right)=\{\emptyset,\{a\}\}$. Since $\mathbf{B}(M(R))=\{\{a, b\}$, $\{a, c\}\}$, so $\mathbf{I}(M(R))=\{\emptyset,\{a\},\{b\},\{c\},\{a, b\},\{a, c\}\}, M(R) \mid X=$ $\left(X, \mathbf{I}_{X}\right),\{c\} \in \mathbf{B}(M(R) \mid X)$, where $\mathbf{I}_{X}=\{\emptyset,\{b\},\{c\}\}$ and $\mathbf{B}(M(R) \mid X)=\{\{b\},\{c\}\}$. Hence, $\mathbf{I}(M(R) / X)=\{\emptyset,\{a\}\}$. Therefore, $\mathbf{I}(M(R) / X)=\mathbf{I}\left(M\left[A_{R} \ominus R^{*}(X)\right]\right)$.

\section{Axioms of Circuit Incidence Matrices of 2-Circuit Matroids}

This section presents two axioms of circuit incidence matrices of 2-circuit matroids. Based on the characteristics of the matroid induced by an equivalence relation, a type of matroids is abstracted, which is called 2-circuit matroid. Therefore, the study of 2-circuit matroid is a further study for the matroid induced by an equivalence relation.

Definition 42 (2-circuit matroid [29]). Let $M=(U, \mathbf{I})$ be a matroid. $M$ is called a 2 -circuit matroid if $|C|=2$ for all $C \in$ $\mathrm{C}(M)$.

Note that if $\mathbf{C}(M)=\emptyset$, then $M$ is also a 2-circuit matroid. In this section, we do not consider this case. The following proposition shows that the matroid induced by an equivalence relation is a 2 -circuit matroid.

Proposition 43 (see [29]). Let $R$ be an equivalence relation on $U$. Then $M(R)$ is a 2-circuit matroid.

In order to obtain the axioms of circuit incidence matrices of 2-circuit matroids, some fundamental signs are presented. Let $\alpha=\left(a_{1}, \ldots, a_{n}\right)$ be a $n$-dimensional $0-1$ vector. Then the complement of $\alpha$ is denoted by $\bar{\alpha}=\left(b_{1}, \ldots, b_{n}\right)$, where

$$
b_{i}= \begin{cases}1, & a_{i}=0 \\ 0, & a_{i}=1 .\end{cases}
$$

According to Definition $20, \varphi^{-1}$ is also a bijection, where $\varphi^{-1}$ is called $\varphi$ inverse. The following lemma shows some properties of $\varphi^{-1}$.

Lemma 44 (see [36]). Let $\alpha$ and $\beta$ be n-dimensional 0-1 vectors and $\varphi$ the characteristic function from $2^{U}$ to the set of all $n$-dimensional 0-1 vectors. Then the following conditions hold:

(1) $\varphi^{-1}(\alpha \wedge \beta)=\varphi^{-1}(\alpha) \cap \varphi^{-1}(\beta)$;

(2) $\varphi^{-1}(\alpha \vee \beta)=\varphi^{-1}(\alpha) \cup \varphi^{-1}(\beta)$;

(3) $\varphi^{-1}(\bar{\alpha})=U-\varphi^{-1}(\alpha)$.

We shall denote $\alpha \otimes \beta=\alpha \wedge \bar{\beta}$. Hence, $\varphi^{-1}(\alpha \otimes \beta)=$ $\varphi^{-1}(\alpha \wedge \bar{\beta})=\varphi^{-1}(\alpha) \bigcap\left(U-\varphi^{-1}(\beta)\right)=\varphi^{-1}(\alpha)-\varphi^{-1}(\beta)$. The vector whose entries are all 0 is denoted by $\mathbf{0}$. The following theorem presents an axiom of circuit incidence matrices of 2 -circuit matroids through row vectors.

Theorem 45. The matrix

$$
C_{m \times n}=\left(\begin{array}{c}
\alpha_{1} \\
\vdots \\
\alpha_{i} \\
\vdots \\
\alpha_{m}
\end{array}\right)
$$

is a circuit incidence matrix of a 2-circuit matroid if and only if the following conditions hold:

(1) $C_{m \times n}$ is a 0-1 matrix where there are two 1 in any row and any row is different;

(2) if $\alpha_{i} \wedge \alpha_{j} \neq \mathbf{0}$ and $i \neq j$, then there exists $\alpha_{k}$ such that $\alpha_{k}=\left(\alpha_{i} \vee \alpha_{j}\right) \otimes\left(\alpha_{i} \wedge \alpha_{j}\right)$.

Proof. $(\Rightarrow)$ : (1) Since $C_{m \times n}$ is a circuit incidence matrix of a 2-circuit matroid, so there are two 1 in any row of $C_{m \times n}$ and $C_{m \times n}$ is a $0-1$ matrix. According to (C2) of Proposition 11, any row of $C_{m \times n}$ is different.

(2) If $\alpha_{i} \wedge \alpha_{j} \neq \mathbf{0}$ and $i \neq j$, then there exist $C_{i}, C_{j} \in \mathbf{C}$, $C_{i} \neq C_{j}$, and $x \in C_{i} \cap C_{j}$, where $C_{i}=\varphi^{-1}\left(\alpha_{i}\right)$ and $C_{j}=$ $\varphi^{-1}\left(\alpha_{j}\right)$. According to (C3) of Proposition 11, there exists $C_{k} \in$ C such that $C_{k} \subseteq\left(C_{i} \cup C_{j}\right)-\{x\}$. Since $\left|C_{i}\right|=\left|C_{j}\right|=\left|C_{k}\right|=2$, so $\{x\}=C_{i} \cap C_{j}$ and $C_{k}=\left(C_{i} \cup C_{j}\right)-\{x\}$. Hence, there exists $\alpha_{k}=\varphi\left(C_{k}\right)$ such that $\varphi^{-1}\left(\alpha_{k}\right)=\left(\varphi^{-1}\left(\alpha_{i}\right) \bigcup \varphi^{-1}\left(\alpha_{j}\right)\right)-$ $\left(\varphi^{-1}\left(\alpha_{i}\right) \cap \varphi^{-1}\left(\alpha_{j}\right)\right)=\varphi^{-1}\left(\alpha_{i} \vee \alpha_{j}\right)-\varphi^{-1}\left(\alpha_{i} \wedge \alpha_{j}\right)=\varphi^{-1}\left[\left(\alpha_{i} \vee\right.\right.$ $\left.\alpha_{j}\right) \otimes\left(\alpha_{i} \wedge \alpha_{j}\right)$ ]. Since $\varphi^{-1}$ is a bijection, so $\alpha_{k}=\left(\alpha_{i} \vee \alpha_{j}\right) \otimes$ $\left(\alpha_{i} \wedge \alpha_{j}\right)$.

$(\Leftarrow)$ : We need to prove that $\left\{\varphi^{-1}\left(\alpha_{i}\right): 1 \leq i \leq m\right\}$ satisfies (C1), (C2), and (C3) of Proposition 11 and $\left|\varphi^{-1}\left(\alpha_{i}\right)\right|=$ 2 for any $1 \leq i \leq m$. Since $C_{m \times n}$ is a $0-1$ matrix where there are two 1 in any row and any row is different, so it is straightforward that $\left\{\varphi^{-1}\left(\alpha_{i}\right): 1 \leq i \leq m\right\}$ satisfies (C1) and (C2), and $\left|\varphi^{-1}\left(\alpha_{i}\right)\right|=2$ for any $1 \leq i \leq m$. Since $\alpha_{i} \wedge \alpha_{j} \neq \mathbf{0}$ and $i \neq j$, so $\varphi^{-1}\left(\alpha_{i}\right) \neq \varphi^{-1}\left(\alpha_{j}\right)$ and $x \in$ $\varphi^{-1}\left(\alpha_{i}\right) \bigcap \varphi^{-1}\left(\alpha_{j}\right)$. Since $\left|\varphi^{-1}\left(\alpha_{i}\right)\right|=2$ for any $1 \leq i \leq m$, so $\varphi^{-1}\left(\alpha_{i}\right) \cap \varphi^{-1}\left(\alpha_{j}\right)=\{x\}$. Then there exists $\varphi^{-1}\left(\alpha_{k}\right)$ such that $\varphi^{-1}\left(\alpha_{k}\right)=\varphi^{-1}\left[\left(\alpha_{i} \vee \alpha_{j}\right) \otimes\left(\alpha_{i} \wedge \alpha_{j}\right)\right]=\varphi^{-1}\left(\alpha_{i} \vee \alpha_{j}\right)-$ $\varphi^{-1}\left(\alpha_{i} \wedge \alpha_{j}\right)=\left(\varphi^{-1}\left(\alpha_{i}\right) \bigcup \varphi^{-1}\left(\alpha_{j}\right)\right)-\left(\varphi^{-1}\left(\alpha_{i}\right) \bigcap \varphi^{-1}\left(\alpha_{j}\right)\right)=$ $\left(\varphi^{-1}\left(\alpha_{i}\right) \cup \varphi^{-1}\left(\alpha_{j}\right)\right)-\{x\}$. Hence, $\left\{\varphi^{-1}\left(\alpha_{i}\right): 1 \leq i \leq m\right\}$ satisfies (C3) of Proposition 11. This completes the proof.

The following lemma shows how a matroid induces an equivalence relation through circuits of the matroid.

Lemma 46 (see [21]). Let $M=(U, \mathbf{I})$ be a matroid. For any $x, y \in U$, one can denote a relation $R$ such that $x R y$ if and only if $x=y$ or $\{x, y\} \in \mathbf{C}(M)$. Then $R$ is an equivalence relation.

The following theorem presents another axiom of circuit incidence matrices of 2-circuit matroids through row vectors and a special relation. 
Theorem 47. The matrix

$$
C_{m \times n}=\left(\begin{array}{c}
\alpha_{1} \\
\vdots \\
\alpha_{i} \\
\vdots \\
\alpha_{m}
\end{array}\right)
$$

is a circuit incidence matrix of a 2-circuit matroid if and only if the following conditions hold:

(1) $C_{m \times n}$ is a 0-1 matrix where there are two 1 in any row and any row is different;

(2) let $x, y \in U, x R y$ if and only if $x=y$ or $\{x, y\} \in$ $\left\{\varphi^{-1}\left(\alpha_{i}\right): 1 \leq i \leq m\right\}$. Then $R$ is an equivalence relation.

Proof. $(\Rightarrow)$ : According to the proof of Theorem 45, we need to prove only the second condition. According to Lemma 46, $R$ is an equivalence relation.

$(\Leftarrow)$ : We need to prove that $\left\{\varphi^{-1}\left(\alpha_{i}\right): 1 \leq i \leq m\right\}$ satisfies (C1), (C2), and (C3) of Proposition 11 and $\left|\varphi^{-1}\left(\alpha_{i}\right)\right|=$ 2 for any $1 \leq i \leq m$. Since $C_{m \times n}$ is a $0-1$ matrix where there are two 1 in any row and any row is different, so it is straightforward that $\left\{\varphi^{-1}\left(\alpha_{i}\right): 1 \leq i \leq m\right\}$ satisfies (C1) and (C2), and $\left|\varphi^{-1}\left(\alpha_{i}\right)\right|=2$ for any $1 \leq i \leq m$. Let $\varphi^{-1}\left(\alpha_{h}\right), \varphi^{-1}\left(\alpha_{j}\right) \in\left\{\varphi^{-1}\left(\alpha_{i}\right): 1 \leq i \leq m\right\}, \varphi^{-1}\left(\alpha_{h}\right) \neq \varphi^{-1}\left(\alpha_{j}\right)$, and $x \in \varphi^{-1}\left(\alpha_{h}\right) \cap \varphi^{-1}\left(\alpha_{j}\right)$. Since $\left|\varphi^{-1}\left(\alpha_{h}\right)\right|=\left|\varphi^{-1}\left(\alpha_{j}\right)\right|=2$, so $\varphi^{-1}\left(\alpha_{h}\right) \cap \varphi^{-1}\left(\alpha_{j}\right)=\{x\}$. Suppose $\varphi^{-1}\left(\alpha_{h}\right)=\{x, y\}$ and $\varphi^{-1}\left(\alpha_{j}\right)=\{x, z\}$, where $x, y, z \in U$ and $x \neq y \neq z$. Therefore, $x R y$ and $x R z$. Since $R$ is an equivalence relation, so $y R z$. Therefore, there exists $\varphi^{-1}\left(\alpha_{k}\right) \in\left\{\varphi^{-1}\left(\alpha_{i}\right): 1 \leq i \leq m\right\}$ such that $\varphi^{-1}\left(\alpha_{k}\right)=\{y, z\}$; that is, $\varphi^{-1}\left(\alpha_{k}\right)=\left(\varphi^{-1}\left(\alpha_{h}\right) \bigcup \varphi^{-1}\left(\alpha_{j}\right)\right)-$ $\{x\}$. Hence, $\left\{\varphi^{-1}\left(\alpha_{i}\right): 1 \leq i \leq m\right\}$ satisfies $(C 3)$ of Proposition 11. This completes the proof.

Note that Theorems 45 and 47 show us a new view to define matroids through circuit incidence matrices. There is not an axiom about circuit incidence matrices in matroid theory and there are three conditions in the circuit axiom, but there are only two conditions in the axiom of circuit incidence matrices of 2-circuit matroids.

\section{Conclusions}

In this paper, we apply matrices to a matroidal structure of rough sets. We prove that the matroid induced by an equivalence relation on a universe is a representable matroid. Some characteristics of the matroid are presented through the representable matrix of the matroid mainly. We apply contraction and restriction operations to the matroid through the representable matrix and approximation operators of rough sets. Moreover, two axioms of circuit incidence matrices of 2 -circuit matroids are obtained. We will do more works in combining rough sets and matroids through matrices.

\section{Acknowledgments}

This work is supported in part by the National Natural Science Foundation of China under Grant nos. 61170128, 61379049, and 61379089, the Natural Science Foundation of Fujian Province, China, under Grant no. 2012J01294, the Science and Technology Key Project of Fujian Province, China, under Grant no. 2012H0043, and the Zhangzhou Research Fund under Grant no. Z2011001.

\section{References}

[1] Z. Pawlak, Rough Sets: Theoretical Aspects of Reasoning about Data, Kluwer Academic Publishers, Boston, Mass, USA, 1991.

[2] Z. Pawlak, "Rough sets," International Journal of Computer and Information Sciences, vol. 11, no. 5, pp. 341-356, 1982.

[3] D. Chen, C. Wang, and Q. Hu, "A new approach to attribute reduction of consistent and inconsistent covering decision systems with covering rough sets," Information Sciences, vol. 177, no. 17, pp. 3500-3518, 2007.

[4] X. Jia, W. Liao, Z. Tang, and L. Shang, "Minimum cost attribute reduction in decision-theoretic rough set models," Information Sciences, vol. 219, pp. 151-167, 2013.

[5] Y. Chen, D. Miao, R. Wang, and K. Wu, "A rough set approach to feature selection based on power set tree," Knowledge-Based Systems, vol. 24, no. 2, pp. 275-281, 2011.

[6] M. Dash and H. Liu, "Consistency-based search in feature selection," Artificial Intelligence, vol. 151, no. 1-2, pp. 155-176, 2003.

[7] Q. Hu, D. Yu, J. Liu, and C. Wu, "Neighborhood rough set based heterogeneous feature subset selection," Information Sciences, vol. 178, no. 18, pp. 3577-3594, 2008.

[8] J. Dai, W. Wang, Q. Xu, and H. Tian, "Uncertainty measurement for interval-valued decision systems based on extended conditional entropy," Knowledge-Based Systems, vol. 27, pp. 443-450, 2012.

[9] Y. Du, Q. Hu, P. Zhu, and P. Ma, "Rule learning for classification based on neighborhood covering reduction," Information Sciences, vol. 181, no. 24, pp. 5457-5467, 2011.

[10] S. K. Pal, S. Mitra, and P. Mitra, "Rough-fuzzy MLP: modular evolution, rule generation, and evaluation," IEEE Transactions on Knowledge and Data Engineering, vol. 15, no. 1, pp. 14-25, 2003.

[11] X. Wang, E. C. C. Tsang, S. Zhao, D. Chen, and D. S. Yeung, "Learning fuzzy rules from fuzzy samples based on rough set technique," Information Sciences, vol. 177, no. 20, pp. 4493-4514, 2007.

[12] J. A. Johnson, M. Liu, and H. Chen, "Unification of knowledge discovery and data mining using rough sets approach in a real-world application," in Rough Sets and Current Trends in Computing, vol. 2005, pp. 330-337, Springer, 2001.

[13] Y. Leung, T. Fung, J. Mi, and W. Wu, "A rough set approach to the discovery of classification rules in spatial data," International Journal of Geographical Information Science, vol. 21, pp. 10331058, 2007.

[14] N. Zhong, "Rough sets in knowledge discovery and data mining," Journal of Japan Society for Fuzzy Theory and Systems, vol. 13, pp. 581-591, 2001.

[15] M. Kryszkiewicz, "Rough set approach to incomplete information systems," Information Sciences, vol. 112, no. 1-4, pp. 39-49, 1998. 
[16] K. Qin, J. Yang, and Z. Pei, "Generalized rough sets based on reflexive and transitive relations," Information Sciences, vol. 178, no. 21, pp. 4138-4141, 2008.

[17] S. Wang, Q. Zhu, W. Zhu, and F. Min, "Quantitative analysis for covering-based rough sets through the upper approximation number," Information Sciences, vol. 220, pp. 483-491, 2013.

[18] W. Zhu and F.-Y. Wang, "Reduction and axiomization of covering generalized rough sets," Information Sciences, vol. 152, pp. 217-230, 2003.

[19] Z. Meng and Z. Shi, "A fast approach to attribute reduction in incomplete decision systems with tolerance relation-based rough sets," Information Sciences, vol. 179, no. 16, pp. 2774-2793, 2009.

[20] Y. Qian, J. Liang, W. Pedrycz, and C. Dang, "Positive approximation: an accelerator for attribute reduction in rough set theory," Artificial Intelligence, vol. 174, no. 9-10, pp. 597-618, 2010.

[21] H. Lai, Matroid Theory, Higher Education Press, Beijing, China, 2001.

[22] Y. Li, Some researches on fuzzy matroids [Ph.D. thesis], Shaanxi Normal University, 2007.

[23] J. Oxley, Matroid Theory, vol. 21 of Oxford Graduate Texts in Mathematics, Oxford University Press, Oxford, UK, 2nd edition, 2011.

[24] J. Edmonds, "Matroids and the greedy algorithm," Mathematical Programming, vol. 1, pp. 127-136, 1971.

[25] E. Lawler, Combinatorial Optimization: Networks and Matroids, Dover, Mineola, NY, USA, 2001.

[26] S. El Rouayheb, A. Sprintson, and C. Georghiades, "On the index coding problem and its relation to network coding and matroid theory," IEEE Transactions on Information Theory, vol. 56, no. 7, pp. 3187-3195, 2010.

[27] R. Dougherty, C. Freiling, and K. Zeger, "Networks, matroids, and non-Shannon information inequalities," IEEE Transactions on Information Theory, vol. 53, no. 6, pp. 1949-1969, 2007.

[28] X. Li and S. Liu, "Matroidal approaches to rough sets via closure operators," International Journal of Approximate Reasoning, vol. 53, no. 4, pp. 513-527, 2012.

[29] S. Wang, Q. Zhu, W. Zhu, and F. Min, "Matroidal structure of rough sets and its characterization to attribute reduction," Knowledge-Based Systems, vol. 36, pp. 155-161, 2012.

[30] W. Zhu and S. Wang, "Matroidal approaches to generalized rough sets based on relations," International Journal of Machine Learning and Cybernetics, vol. 2, no. 4, pp. 273-279, 2011.

[31] W. Zhu and S. Wang, "Rough matroids based on relations," Information Sciences, vol. 232, pp. 241-252, 2013.

[32] H. Mao, "Relations between matroids and concept lattices," Advances in Mathematics (China). Shuxue Jinzhan, vol. 35, no. 3, pp. 361-365, 2006.

[33] F. Matúš, "Abstract functional dependency structures," Theoretical Computer Science, vol. 81, no. 1, pp. 117-126, 1991.

[34] Y. Y. Yao, "Relational interpretations of neighborhood operators and rough set approximation operators," Information Sciences, vol. 111, no. 1-4, pp. 239-259, 1998.

[35] Y. Y. Yao, "Constructive and algebraic methods of the theory of rough sets," Information Sciences, vol. 109, no. 1-4, pp. 21-47, 1998.

[36] R. Johnsonbaugh, Discrete Mathematics, Publishing House of Electronics Industry, 2009.

[37] J. Wang and W. Zhu, "Contraction to matroidal structure of rough sets," in Rough Sets and Knowledge Technology, vol. 8171 of Lecture Notes in Computer Science, pp. 75-86, Springer, 2013. 


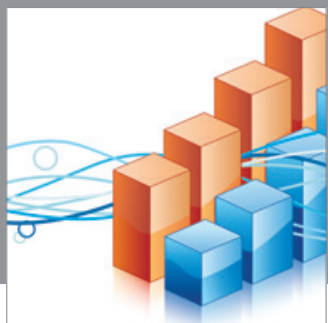

Advances in

Operations Research

mansans

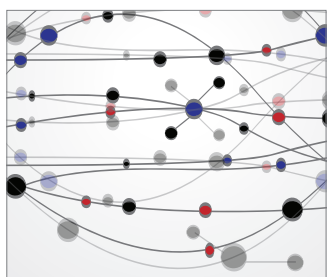

The Scientific World Journal
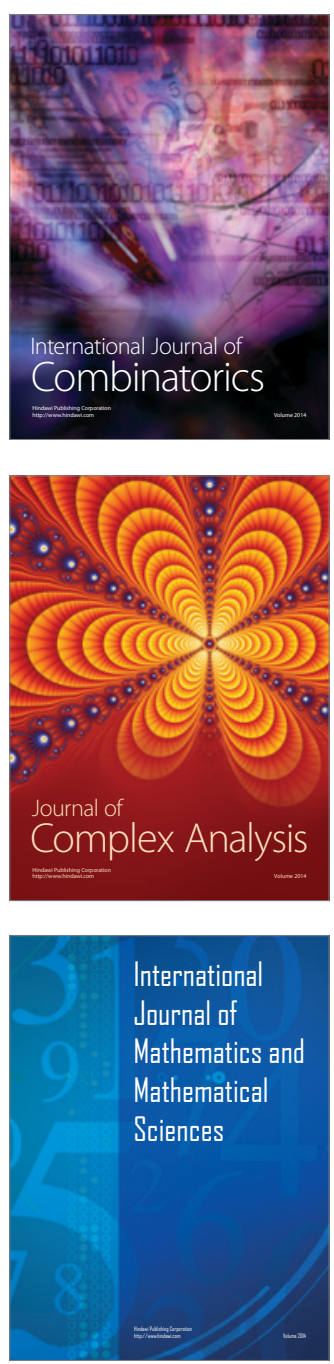
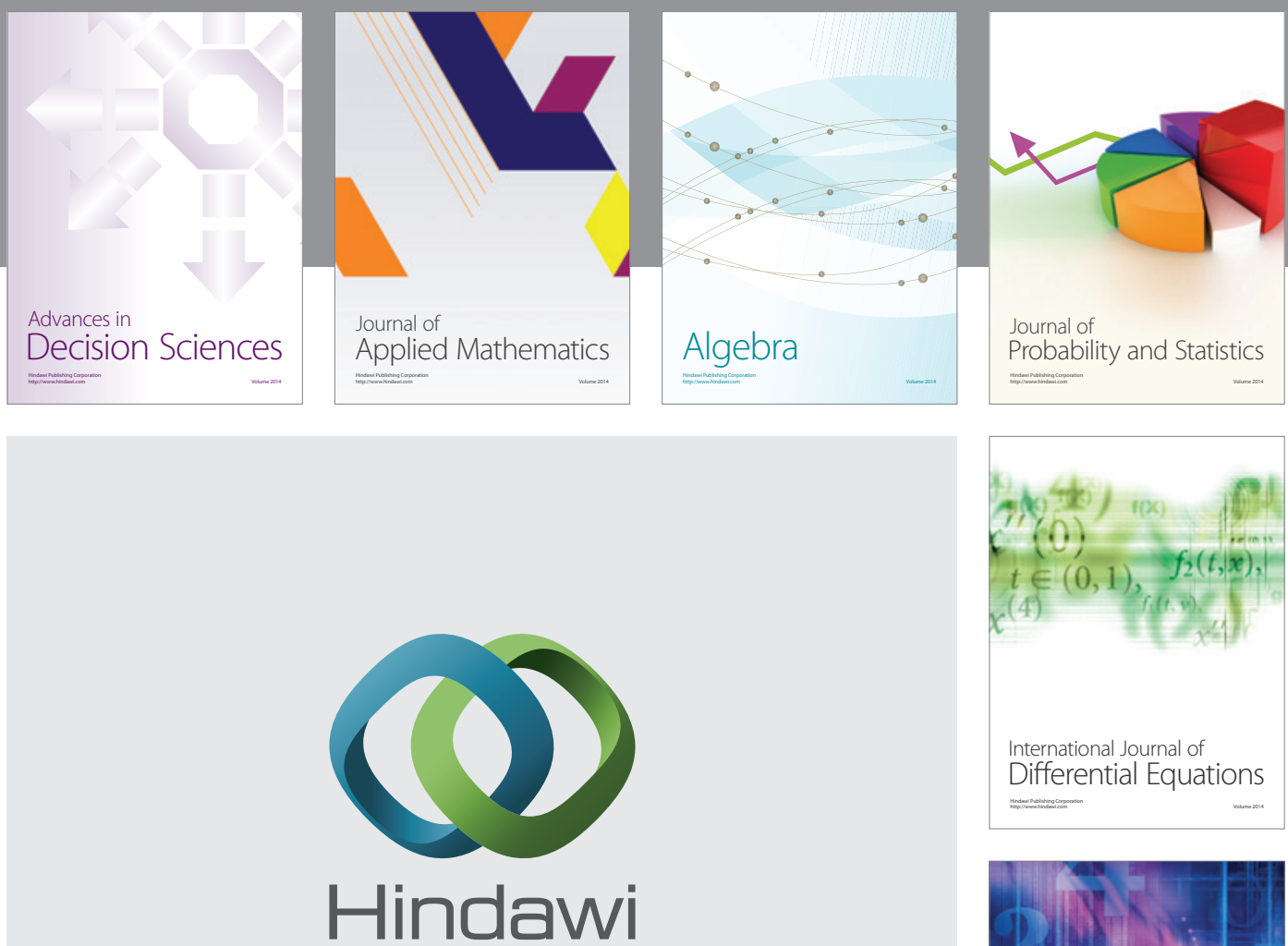

Submit your manuscripts at http://www.hindawi.com
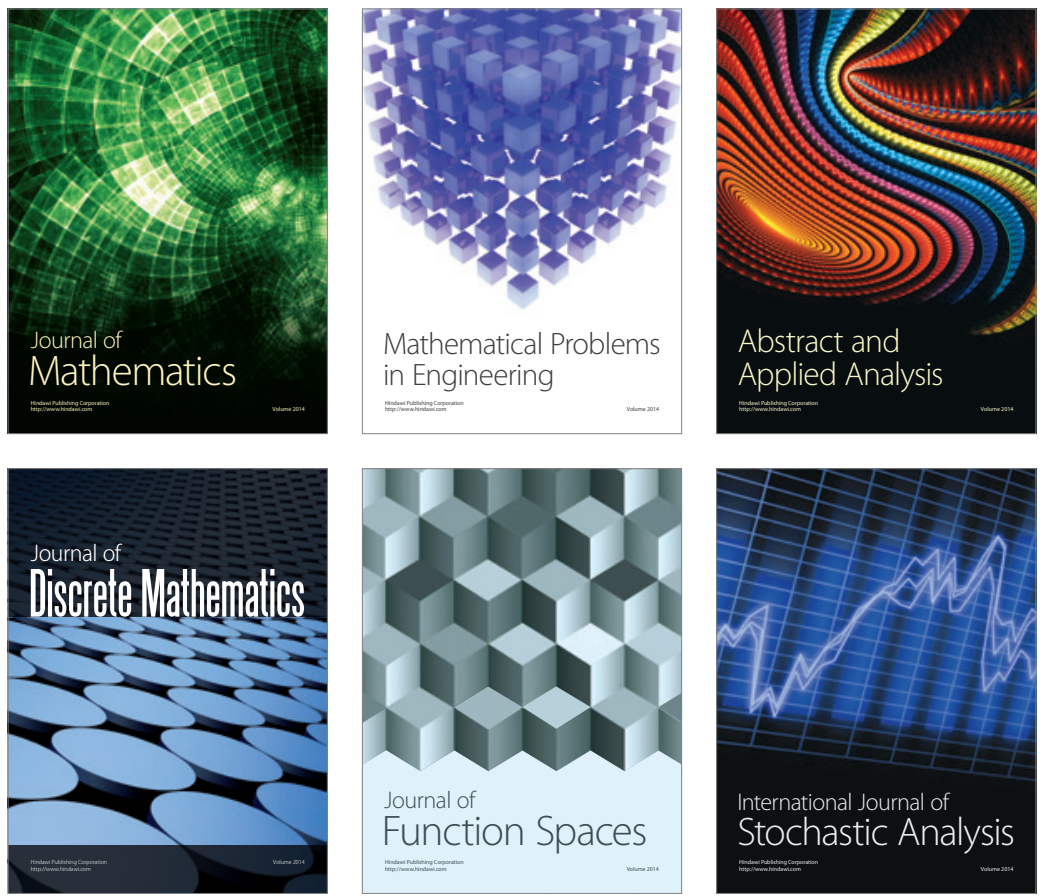

Journal of

Function Spaces

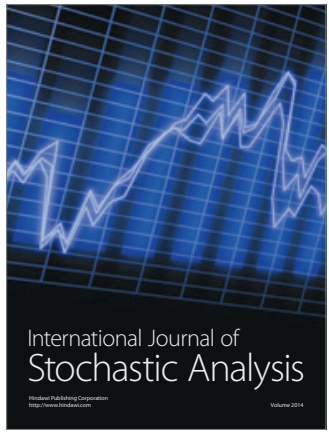

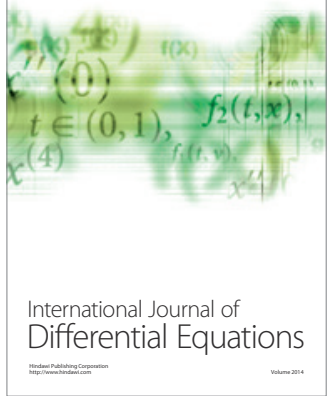
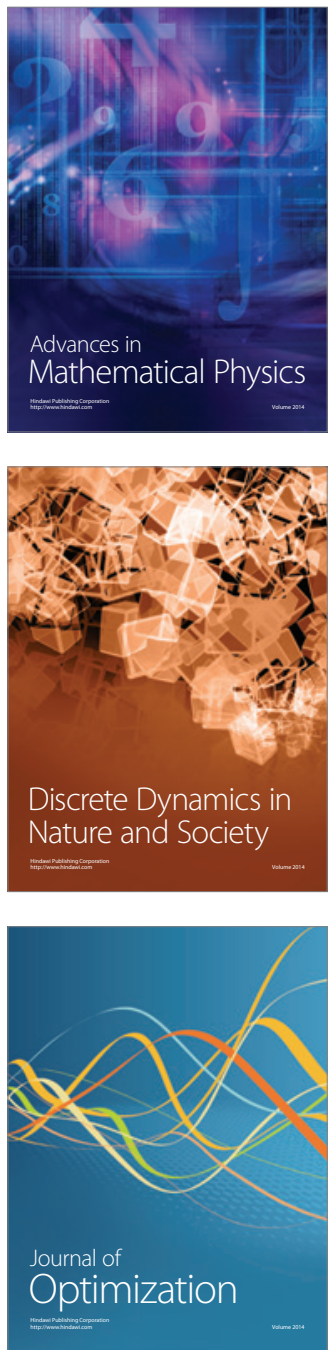